\title{
Aero-engine Casings Modal Characteristic Assessment Using an Efficenint and Novel Modal Assurance Criterion Methodology
}

\author{
Seyed-Ehsan Mir-Haidari ${ }^{1}$, Kamran Behdinan ${ }^{2}$ \\ ${ }^{1}$ Department of Mechanical and Industrial Engineering, University of Toronto, Toronto, Canada \\ ${ }^{2}$ Department of Mechanical and Industrial Engineering, University of Toronto, Toronto, Canada \\ 1ehsan.mirheidaru@mail.utoronto.ca \\ ${ }^{2}$ Behdinan@mie.utoronto.ca
}

\begin{abstract}
In this paper, a novel modelling methodology and experimental protocol has been proposed for a computationally efficient means of performing modal characteristic assessment while addressing the issue of spatial phase angle difference that arises in vibration analysis of axisymmetric structures. An experimental modal characteristic assessment is performed by means of a roving hammer impact test, frequency comparison, and a novel modal assurance criterion assessment methodology. The technical accuracy of the obtained vibrational characteristics is verified using simulations results based on both high fidelity and simplified FEM models. LMS software is used to perform the experimental assessment and post processing of data while MSC. PATRAN and MSC.NASTRAN are used to implement the proposed novel theory of addressing spatial phase angle differences that are associated to axisymmetric structures, such as the aero-engine casings.
\end{abstract}

Keywords-Aero-engines; modal assessment; vibration analysis; axisymmetric structures; modal assurance criteria; FEM; natural frequency

\section{INTRODUCTION}

Aero-engine rotor induced vibration is the primary source of structural vibration in the aircraft, which is primarily attributed to mass unbalance loadings in the rotor system. The extent of unbalance loadings in the aero-engine is directly related to the extent of mass eccentricity in the system [1], which is caused by imperfections in the manufacturing process of the aeroengine rotor system [2]. The unbalance loadings in the aeroengine is a prime contributor of fuselage vibration, effecting the passenger and crew's comfort in the aircraft cabin. Considering advancements in aero-engine design technologies with the overall aim of mass reduction to improve efficiency. Due to these circumstances, vibration reduction and control becomes a paramount goal in the design and development of novel aero-engines [3]

Ground vibration testing (GVT) is an important aspects of aircraft and aero-engine design and development to analyze the dynamic behavior and modal characteristic of various aircraft structures[4]. Obtained empirical data from GVT testing are compared to validate the developed finite element models (FEM) of various aircraft components. This protocol is used to obtain reliable and dependable FEMs to accurately predict the dynamic response (i.e. modal characteristics) of the aircraft components. The GVT is a mandated process that is required for aircraft certification [5]. However, a guideline implemented by the European aero-space requires the minimization of GVT time to minimize the associated testing costs [6]. Therefore, it is significantly important to develop computationally efficient FEM of aircraft components such as the aero-engine to minimize the computational effort as required by GVT, while providing the required accuracy and reliability for certification and validation process.

For aero-engine design and development, Finite Element Analysis is an established tool for vibration and structural analysis [7]-[10]. In order to perform Finite Element Analysis (FEA) for complex geometries, simplified FE models are required in order to accurately capture the representative system dynamics while reducing the computation effort. Due to simplification of the complex geometries, it is inherently important to perform model validation of the developed simplified FE models, including natural frequency and Modal Assurance Criteria (MAC) comparison [11]-[13].

To perform model validation, the measured modal characteristics of the aero-engine casing structures is compared using both theoretical and experimental results and data. As presented in this paper, initially a Roving Hammer Impact Testing is initially performed to obtain the natural frequencies and mode shapes. In the next phase, MAC comparison is performed. MAC is an indicator that is used to calculate and compare similarities between mode shapes [14].

However, MAC suffers from inaccuracies in achieving good correlations for axisymmetric geometries, such as the aeroengine casings due to different spatial phase angles [15]. Aeroengine casings are quasiaxisymmetric structure (due to imperfections that are present on the structures such as holes and extrusions). It should be noted that the actual physical mode will have mode shapes and natural frequencies that are very close to predictions by FEM, yet with minor difference in the spatial angle. The same issue would arise for MAC validation of both high-fidelity and simplified model of the 
aero-engine casings that are required to achieve computational efficiency. It should be noted that visual inspections of the modes would indicate a very close relationship between the modes; however, a MAC comparison would yield low MAC values due to the spatial angle difference [15].

In this paper, a novel and systematic modal characteristic validation process for implementation on axisymmetric structures based on a novel and efficient FE modelling strategy using both high fidelity and simplified FEMs that is implemented on two aero-engine casings to propose a time efficient and accurate certification process to meet the demands of the aerospace industry.

\section{AERO-ENGINE CASINGS FEM}

For complex geometrical structures, it is significantly important to introduce simplified FEMs to significantly reduce the associated computation effort, while achieving the intended accuracy that is required for modal assessment [11][13], [16].

As a benchmark, two novel modelling methodologies to develop high fidelity and simplified FEMs of front and rear aero-engine compressor casings are proposed. The obtained modal characteristics results are compared to validate the results of the simplified FEM. All the FEM presented in the paper are developed using Hyperworks software and all the presented FEMs are created using CQUAD4 elements. The introduced high-fidelity FEM methodology captures all the geometrical features of the aero-engine casing which in turn provides modal characteristics results that are most accurate compared to the empirical data. However, the proposed FEM suffers from a high number of element count (high element density) that could be inefficient in achieving the desired computational efficiency. Development of the high-fidelity FEM is also a very labor-intensive process. It should be emphasized that the goal of this study is to develop a modelling framework that provides the advantage of achieving significant computational efficiency while achieving the intended modal characteristic accuracy. In order to achieve the required computation efficiency to obtain the modal characteristics of the aero-engine structures, the simplified FE methodology has been developed. The simplified FE methodology only captures the important structural topologies on the structure to achieve reduced element count with reduced element density by capturing the axisymmetric features of the structure which enables achieving exceptional computation efficiency compared to the high-fidelity FEM methodology.

The simplified and high-fidelity FEM models of the front and rear compressor casings are presented in Fig. 1 and 2, respectively. The element counts of the high-fidelity FEM of the front and rear compressor casings are approximately 500,000 and 550,000. The simplified FEM of the front and the rear compressor casings are composed of approximately 5,000 and 15,000 elements, respectively. The element count in the simplified FEM has been reduced by a factor of 100 and 37 for the front and rear compressor casing; respectively, compared to the high fidelity FEM. The illustrated modal characteristics results of the natural frequency are normalized using the following equation [17]:

$\omega_{n}=100 \omega R \sqrt{\frac{\rho}{E}}$

where $\omega$ is the natural frequency, $\rho$ is the rear compressor casing's density, $E$ is the rear compressor casing's Young modulus and $R$ is the rear compressor casing's radius (top of the rear compressor casing).

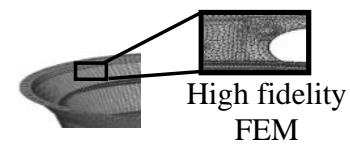

Figure 1- Simplified FEM-Front casing

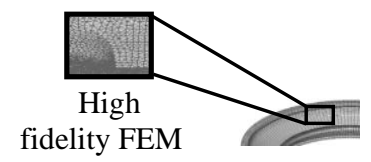

Figure 2- Simplified FEM-Rear casing

To determine the accuracy of the simplified FEM, the natural frequency associated to the high fidelity and simple FEMs are calculated using the MSC.NASTRAN software package. The presented natural frequencies are normalized using the following equation:

Error $\%|\boldsymbol{E}|=\left|\frac{\omega_{\text {Simplified FEM }}-\omega_{\text {High Fidelity FEM }}}{\omega_{\text {High fidelity }}}\right| \times 100 \%$

where $\omega_{\text {High fidelity FEM }}$ is the high fidelity FEM natural frequency and $\omega_{\text {Simplified FEM }}$ is the simplified FEM natural frequency.

\section{MODAL ANALYSIS EXPERIMENTATION FUNDEMENTALS}

As previously mentioned, empirical modal characteristic analysis is critical for assessment of the developed FEMs accuracy. In this experimentation, mode shapes and natural frequencies corresponding to the front and rear compressor casings are determined. The dynamic response of a damped system can be defined by the following equation in the frequency domain:

$$
\mathrm{F}(\mathrm{s})=[\mathbf{M}] s^{2} X+[\mathbf{C}] s X+[\mathbf{K}] X
$$

where $[\mathrm{M}]$ is the mass matric, $[\mathrm{C}]$ is the damping matrix, $[\mathrm{K}]$ is the stiffness matrix, $X$ is the displacement vector and $\mathrm{F}(\mathrm{s})$ is the response vector. The Transfer function of the system can be defined by the following equation:

$\boldsymbol{H}(\mathrm{s})=\left([\mathbf{K}]+[\boldsymbol{C}] \mathrm{s}+[\mathbf{M}] \mathrm{s}^{2}\right)^{-1}$

For a linear structure, total system response can be represented by a combination of single degree of freedom (DOF) systems. This statement implies that the system's 
modal properties can be measured by a constant excitation DOF where responses are measured at any DOF or vice versa.

The frequency response of an entire linear system can be defined by the summation of all single d.o.f.s (degree of freedom). This statement provides a strategy for experimental modal assessment, suggesting that at an excitation input at a single location is enough to measure the response at all other locations or vice versa; the response at single a location can be measured using various excitation locations inputs. These strategies can be used to perform experimental modal characteristic assessment.

\section{EXPERIMENTAL MODAL ANALYSIS SETUP}

It is critical to perform experimental modal characteristic verification of the developed high fidelity and simplified FEM models of the front and rear compressor casings to ensure the credibility and accuracy of FE simulations. As shown in Fig. 4 , the experimental setup consists of an impact hammer for system excitation, accelerometers to measure the aero-engine casings response and a data acquisition package, which is performed by LMS software.

The experimental setup consists of suspending the structure from bungee cords to replicate free-free boundary conditions. A single accelerometer is attached to the casings to measure the responses caused by the impact hammer excitation. The excitation locations consist of fine grid around the casings structure (200 and 90 excitation points on the rear and front casings, respectively) to accurately capture the mode shapes for MAC calculations. Fig. 3 represents the hammer impact testing experimental setup.

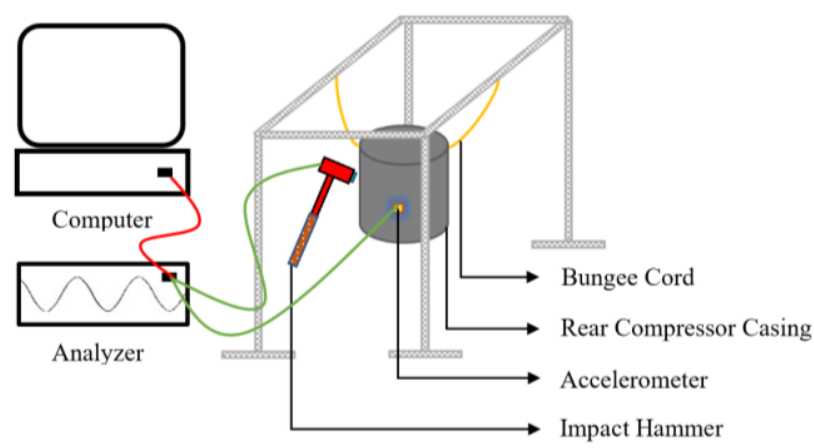

Figure 3- Modal characteristic assessment experimental setup

The difference between the experimental and FEM natural frequencies are calculated using the following equation:

Error $\%|\boldsymbol{E}|=\left|\frac{\omega_{F E M}-\omega_{\text {Exp }}}{\omega_{F F M}}\right| \times 100 \%$

where $\omega_{\text {FEM }}$ and $\omega_{\text {Exp }}$ are the theoretical and experimental natural frequencies of the front and rear compressor casings.

\section{MODAL CHARACTERISTICS CORRELATION METHADOLOGY}

For this paper, the modal characteristic assessment consists of determining the natural frequency and MAC comparison. The analysis will be performed for the frequency range of 0$1000 \mathrm{~Hz}$. The natural frequencies of symmetric structures can be closely separated. Therefore, the best available methodology to determine the correlated mode pairs is by performing MAC calculations. As previously mentioned, due to the axisymmetric nature of casing structures, MAC calculation would erroneous results due to the spatial phase angle differences.

MAC is a commonly used statically indicator that can be used in determining the validity and consistency of FE simulation results and the modal vectors [14], [18]. MAC calculation provides a number that takes a value between 0 and 1 . The value 1 indicates full similarity between the modes shapes and 0 indicates no similarity between the mode shapes. MAC is defined by the following equation [19]:

$\boldsymbol{M A C}(\boldsymbol{E}, \boldsymbol{K})=\frac{\left|\left\{\xi_{Y}\right\}_{E}^{T}\left\{\xi_{v}\right\}_{K}\right|^{2}}{\left(\left\{\xi_{Y}\right\}_{E}^{T}\left\{\xi_{Y}\right\}_{E}\right)\left(\left\{\xi_{v}\right\}_{K}^{T}\left\{\xi_{v}\right\}_{K}\right)}$

Where $\left\{\xi_{v}\right\}_{K}$ is the experimental modal vector (mode $\mathrm{K}$ ), $\left\{\xi_{Y}\right\}_{F}^{T}$ is the transpose of compatible FE modal vector (mode E), $\left\{\xi_{Y}\right\}_{F}$ is the compatible FE modal vector (mode E) and $\left\{\xi_{v}\right\}_{K}^{T}$ is transpose of the experimental modal vector (mode $\mathrm{K}$ ).

Using MAC calculation, the correlated mode pairs can be readily identified and the natural frequency difference between the experimental and theoretical modes can be calculated. To address the issue for axisymmetric structures MAC calculations and spatial angle differences that arise for the correlation between the experimental results and FEM (high fidelity and simplified), a novel MAC calculation protocol has been introduced into NASTRAN, using the script written to NASTRAN. The proposed script generates large pool of simplified FEMs for the paired frequencies using Guyan reduction technique to determine which of the obtained data pool provides highest MAC average. By implementing this technique which utilizes large number of simulations run, it has the capability of finding the right spatial angle that generates the highest MAC value. This procedure was performed on the axisymmetric simplified mesh of the front and rear compressor casings of the aero-engine. The resulting MAC values were determined and are presented as MAC and FMAC plots. The MAC calculation algorithm implemented into NASTRAN using PATRAN command language is represented by the flow chart shown in Fig. 4. 


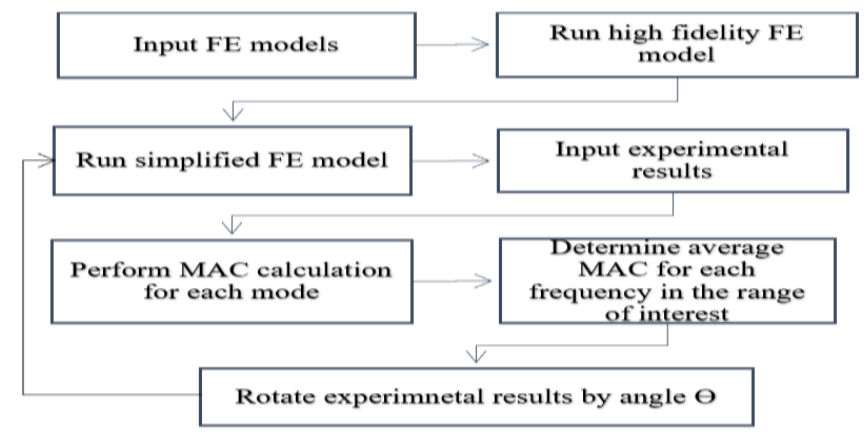

Figure 4- MAC calculation algorithm

\section{RESULTS AND DISCUSSIONS}

As shown by Fig. 5, it can be concluded that that developed and proposed high fidelity and simplified FEM models provide accurate results (all mode pairs have less than $8 \%$ difference) when the natural frequencies are compared to experimentally obtained results. This modal characteristic assessment results provides an indication of the accuracy and validity of the novel and proposed simplified FE meshing methodology in capturing the modal characteristics while achieving exceptional computation efficiency. The computational efficiency achieved using the simplified FEM compared to the high-fidelity FEM resulted in a computational reduction time in the order of 40 for the case modal characteristic assessment. This illustrates the efficiency of proposed methodology in achieving the desired accuracy while being extremely computationally efficient.

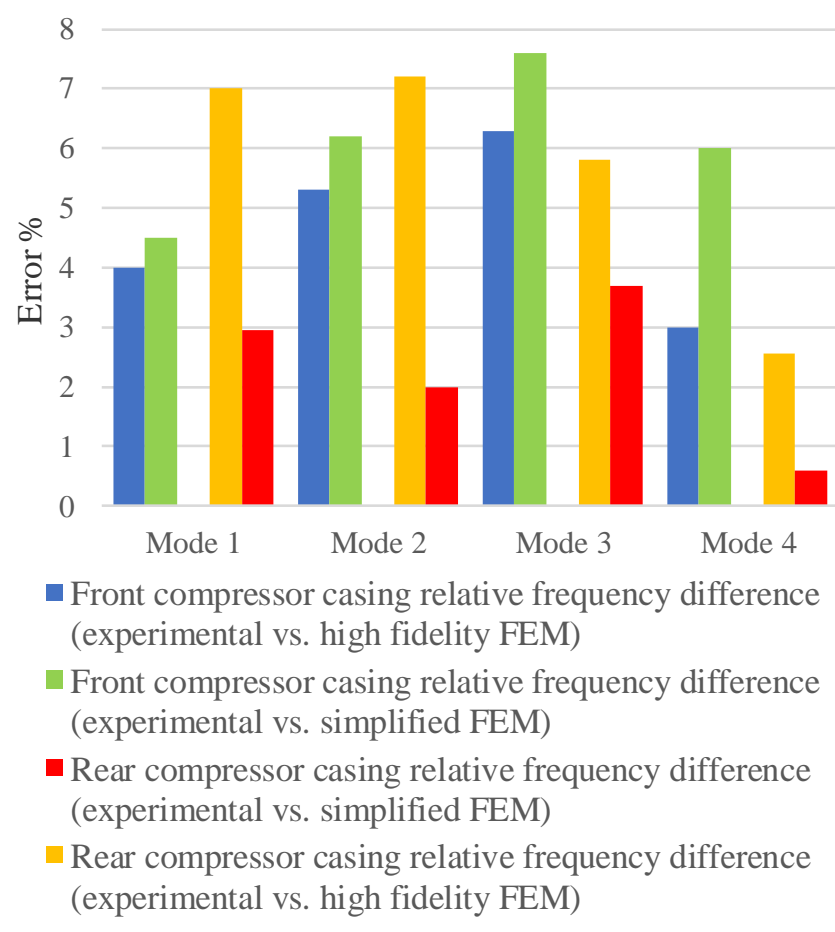

Figure 5- Front and rear casings FEM and experimental natural frequency comparison
FMAC plots for the front and rear casings are presented in Figs. 6-9. The FMAC plot is a novel way of representing combination of information, including MAC, AutoMAC and frequency comparison simultaneously[20]. As seen in Figs. 69 , the regression line is represented by solid purple lines. The MAC values, which is calculated between every possible mode pair is represented on the FMAC plot by various hollow circles with various radiuses (orange circles). The larger radius is an indication of a higher MAC number and better correlation between mode pairs. Moreover, standalone MAC plots are shown in Figs 10-13 for front and rear compressor casings to provide a quantitative MAC value for the correlated mode pairs. The MAC values illustrated in Figs. 10-13 is an illustration of good correlation between experimental results and proposed FEM meshing methodology. By implementing the proposed methodology that addresses the spatial phase angle differences, on average, the MAC values were improved by $20 \%$ for all the correlated mode pairs, which illustrates it importance and significance for consideration in axisymmetric structures. The MAC results also support the validity of the proposed protocol in addressing the issue that arises with axisymmetric features, as shown by the obtained MAC values, they are in the high end of the spectrum (on average, more than 0.9). On the FMAC plots, the AutoMAC values which represents the correlation of the experimental frequencies are represented by filled circles (filled in blue circles). In order to determine the sufficiency of the sampling grids used for experimental testing on the casings, off diagonal AutoMAC values should not be observed on FMAC plot. This is the case for both the rear and front casings, as evident by Figs. 6-9. The shaded area around the solid purple line (regression) represents the $95 \%$ confidence interval for a Gaussian error distribution. For both front and rear compressor casings, the regression line has a slope close to 1 , indicating a very good correlation of theoretical and empirical results for both the high fidelity and simplified FEMs. Overall, the rear casing illustrates better correlation with the empirical results compared to the front compressor casing. This discrepancy can be associated to the observed mass discrepancy that exists between the provided front compressor casing CAD model and the physical casing. In can be concluded that the proposed protocol in addressing and solving different spatial phase angles is a very useful tool and technique for implementation on axisymmetric structures that can be implemented along with the proposed FEM meshing methodology for rapid modal characteristic assessment.

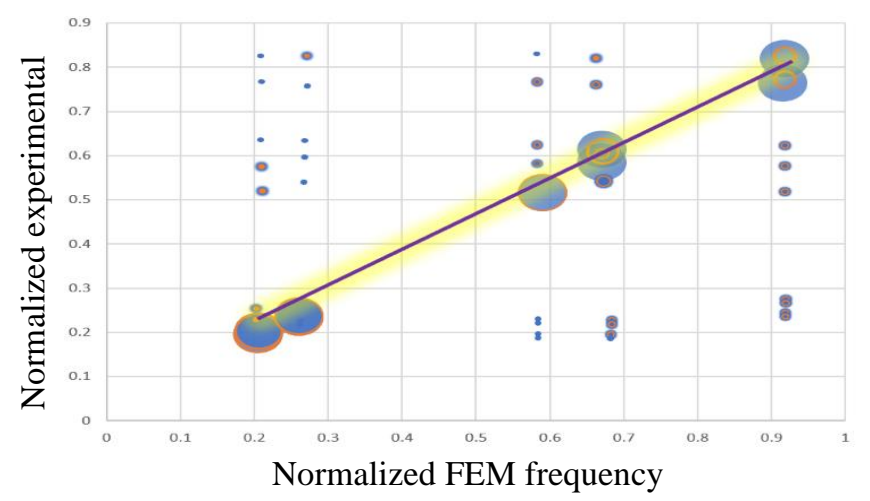

Figure 6-FMAC plot for front casing simplified FEM 


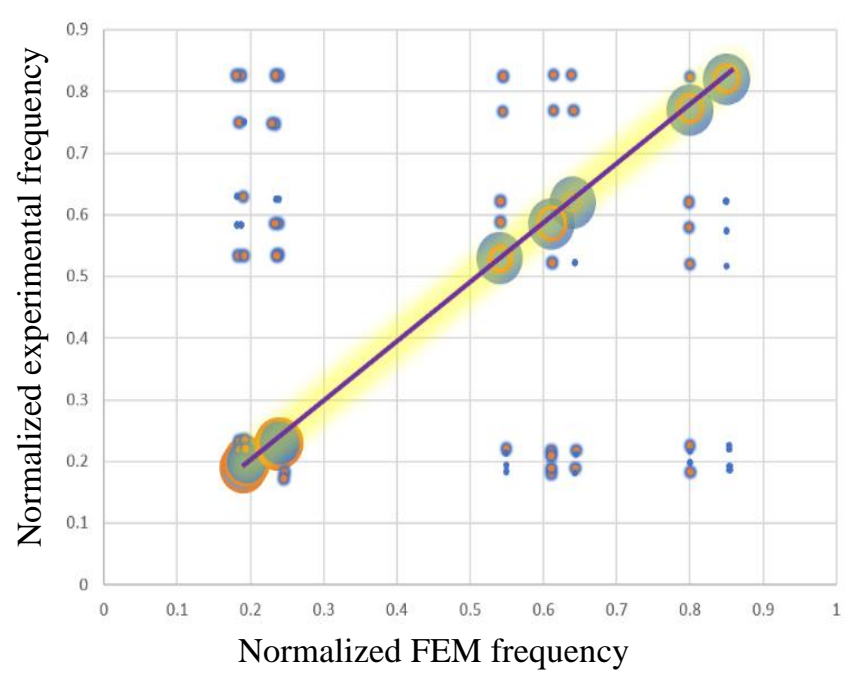

Figure 7- FMAC plot for front casing high fidelity FEM

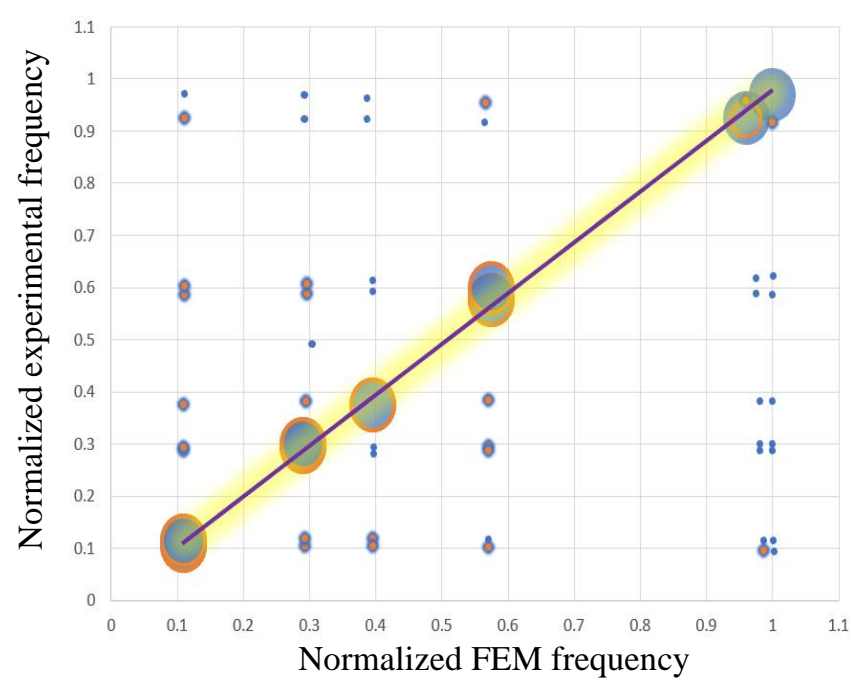

Figure 8-FMAC plot for rear casing simplified FEM

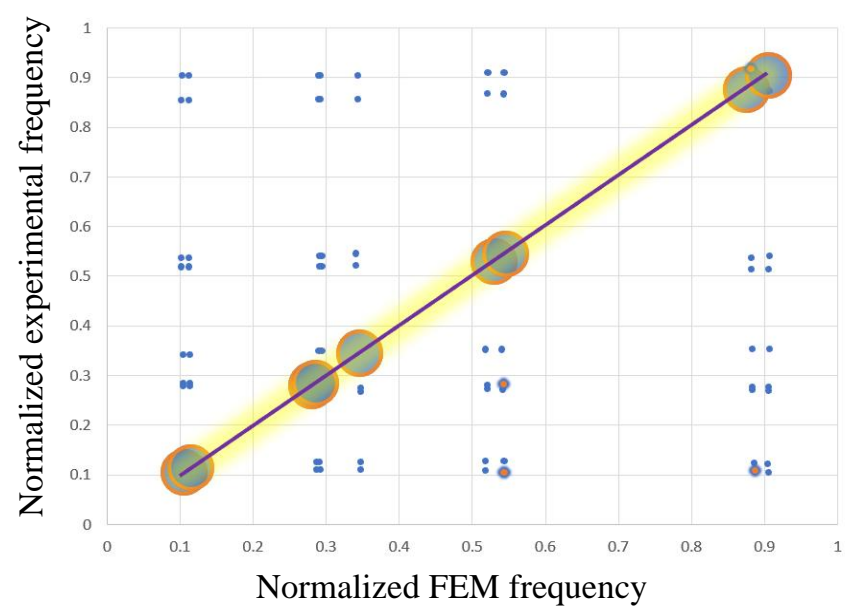

Figure 9- FMAC plot for rear casing high fidelity FEM

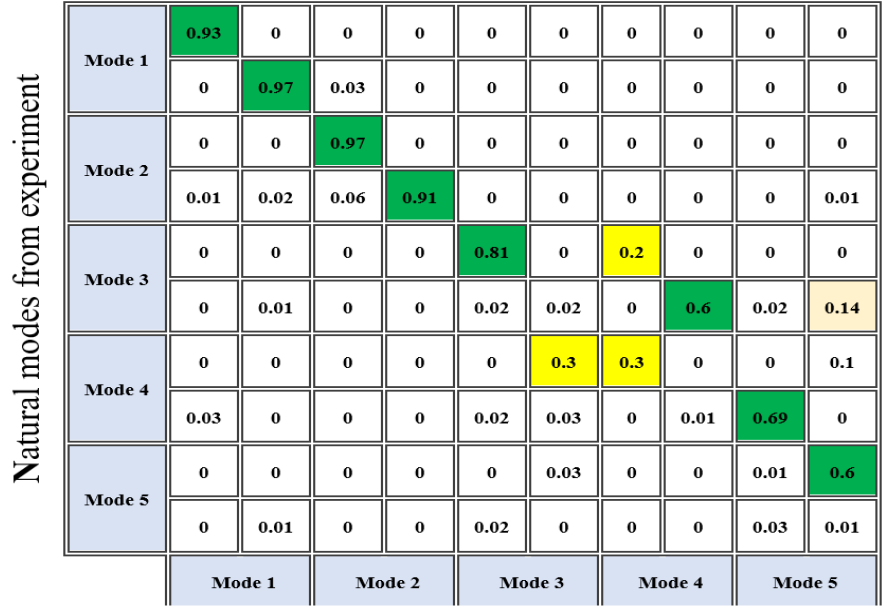

Natural modes from FEM

Figure 10- MAC plot for front casing simplified FEM

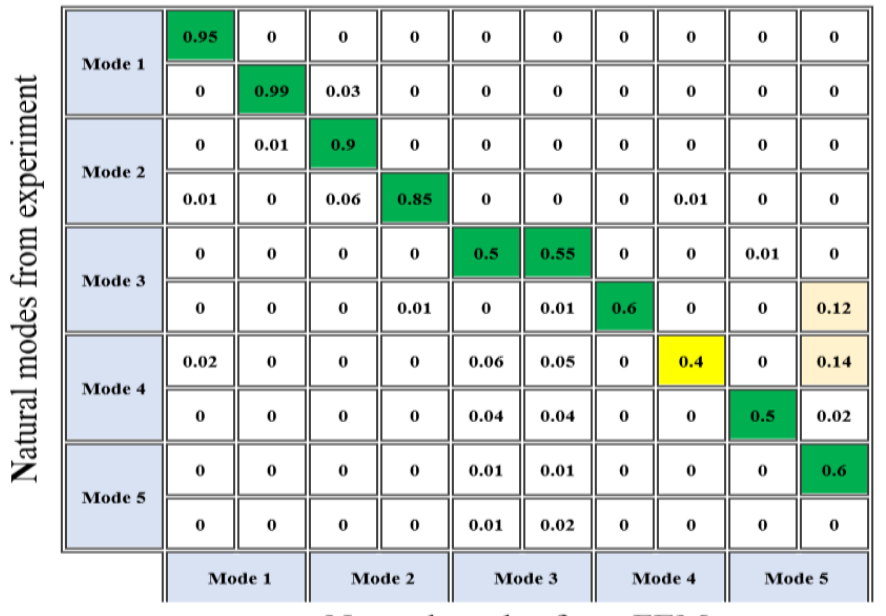

Natural modes from FEM

Figure 11- MAC plot for front casing high fidelity FEM

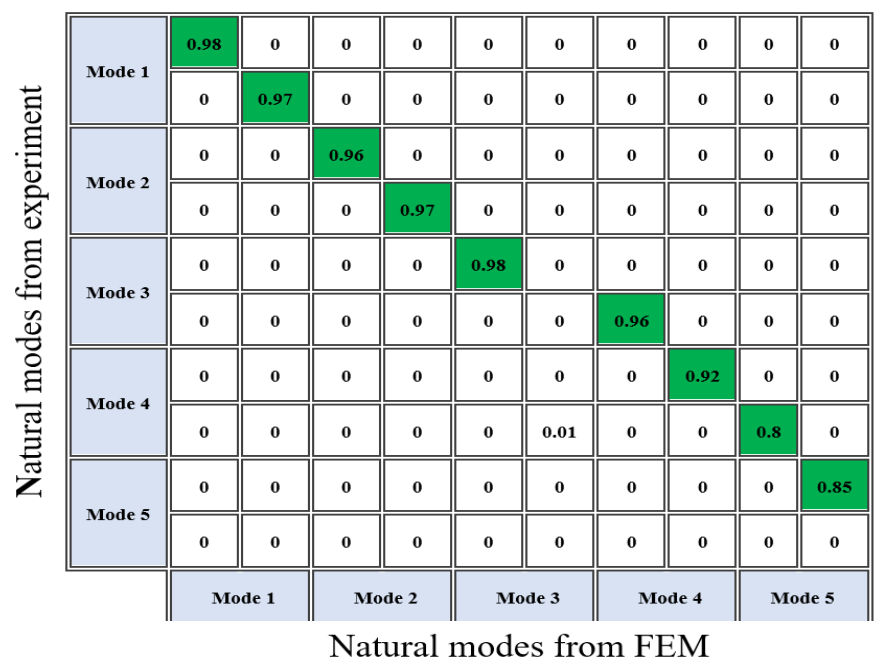

Figure 12- MAC plot for front casing simplified FEM 


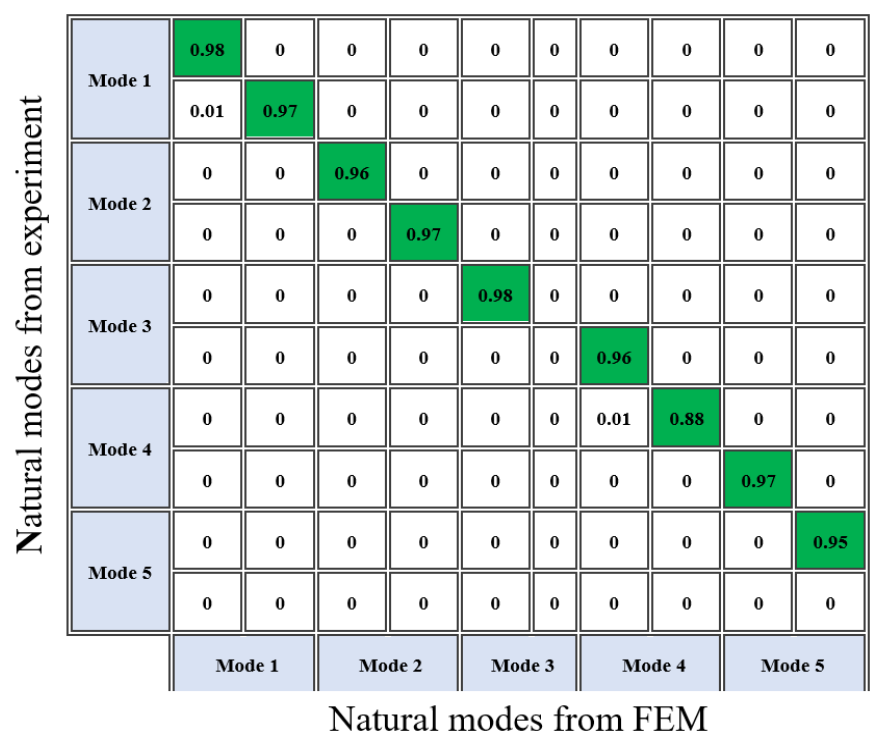

Figure 13- MAC plot for front casing simplified FEM

\section{CONCLUSION}

Hammer impact testing on the front and rear compressor casings provided a validation of accuracy of the proposed methodology in developing simplified FEMs, which was demonstrated to be computationally efficient while being accurate. The frequency comparison between the FEM and experimental results illustrated a difference of less than $8 \%$, validating the overall approach. The AutoMAC values illustrated the validity of the overall experimental approach and setup, as no major outliers were observed. In order to address the spatial phase angle difference, the implemented NASTRAN algorithm allowed obtaining high MAC values for the axisymmetric structure of the aero-engine casings, which was shown to have an overall impact of $20 \%$ increase for all the correlated mode pairs. The FMAC and MAC tables provided accurate and correct related mode pairs with high MAC values and reliable regression lines, laying the ground to confirm the accuracy and reliability of the results associated to the proposed modal assessment methodology.

\section{REFERENCES}

[1] H. M. Navazi and M. Hojjati, "Nonlinear vibrations and stability analysis of a rotor on high-static-low-dynamic-stiffness supports using method of multiple scales," Aerosp. Sci. Technol., vol. 63, pp. 259-265, 2017.
[2] Y. Liu et al., "A method to minimize stage-by-stage initial unbalance in the aero engine assembly of multistage rotors," Aerosp. Sci. Technol., vol. 85, pp. 270-276, 2019.

[3] M. Viscardi and M. Arena, "Sound proofing and thermal properties of an innovative viscoelastic treatment for the turboprop aircraft fuselage," CEAS Aeronaut. J., vol. 10, no. 2, pp. 443-452, 2019.

[4] B. Peeters, J. Debille, and H. Climent, "Modern Solutions for Ground Vibration Testing of Small, Medium and Large Aircraft," SAE Int. J. Aerosp., vol. 1, no. 1, pp. 732-742, 2010.

[5] D. Goege, "Fast Identification and Characterization of Nonlinearities in Experimental Modal Analysis of Large Aircraft," J. Aircr., vol. 44, no. 2, pp. 399-409, 2007.

[6] G. Gloth and M. Sinapius, "Analysis of swept-sine runs during modal identification," Mech. Syst. Signal Process., vol. 18, no. 6, pp. 14211441, 2004.

[7] L. Salles, B. Staples, N. Hoffmann, and C. Schwingshackl, "Continuation techniques for analysis of whole aeroengine dynamics with imperfect bifurcations and isolated solutions," Nonlinear Dyn., vol. 86, no. 3, pp. 1897-1911, 2016.

[8] W. Q. Zhao, Y. X. Liu, and M. W. Lu, "Vibration Analysis of Aeroengine Compressor Blade-Disc Coupling System," Appl. Mech. Mater., vol. 1619, pp. 264-268, 2009.

[9] E. P. Petrov and D. J. Ewins, "State-of-the-art dynamic analysis for nonlinear gas turbine structures," Proc. Inst. Mech. Eng. Part G J. Aerosp. Eng., vol. 218, no. 3, pp. 199-211, 2004.

[10] G. Kerschen, M. Peeters, J. C. Golinval, and C. Stéphan, "Nonlinear Modal Analysis of a Full-Scale Aircraft," J. Aircr., vol. 50, no. 5, pp. 1409-1419, 2013.

[11] G. Banwell, S. Mohr, S. Rothberg, and J. Roberts, "Using experimental modal analysis to validate a finite element model of a tennis racket," Procedia Eng., vol. 34, no. 0, pp. 688-693, 2012.

[12] S. Y. Chen, "An equivalent direct modeling of a rotary shaft with hot-fit components using contact element modal analysis results," Comput. Math. with Appl., vol. 64, no. 5, pp. 1093-1099, 2012.

[13] D. Ribeiro, R. Calçada, R. Delgado, M. Brehm, and V. Zabel, "Finite element model updating of a bowstring-arch railway bridge based on experimental modal parameters," Eng. Struct., vol. 40, pp. 413-435, 2012.

[14] M. Pastor, M. Binda, and T. Harčarik, "Modal assurance criterion," Procedia Eng., vol. 48, pp. 543-548, 2012.

[15] G. Chen, D. Fotsch, N. Imamovic, and D. J. Ewins, "Correlation methods for axisymmetric structures," Proc. Int. Modal Anal. Conf. - IMAC, vol. 2, pp. 1006-1012, 2000.

[16] C. G. Martin and A. Arokkiaswamy, "Finite element modal analysis and mesh optimization of a typical turbo fan engine - fan hub frame," Int. J. Adv. Sci. Res. Manag., vol. 1, no. 3, pp. 95-102, 2016.

[17] R. Moradi-Dastjerdi and H. Momeni-Khabisi, "Vibrational behavior of sandwich plates with functionally graded wavy carbon nanotubereinforced face sheets resting on Pasternak elastic foundation," JVC/Journal Vib. Control, vol. 24, no. 11, pp. 2327-2343, 2018.

[18] R. Pascual, J. C. Golinval, and M. Razeto, "A Frequency Domain Correlation Technique for Model Correlation and Updating," 15th Int. Modal Anal. Conf. (IMAC XV), pp. 1-7, 1997.

[19] R. A. Ibrahim and C. L. Pettit, "Uncertainties and dynamic problems of bolted joints and other fasteners," J. Sound Vib., vol. 279, no. 3-5, pp. 857-936, 2005.

[20] D. W. Fotsch and D. J. Ewins, "Application of Mac in the Frequency Domain,” IMAC-XVIII A Conf. Struct. Dyn., vol. 2, pp. 1225-1231, 2000. 